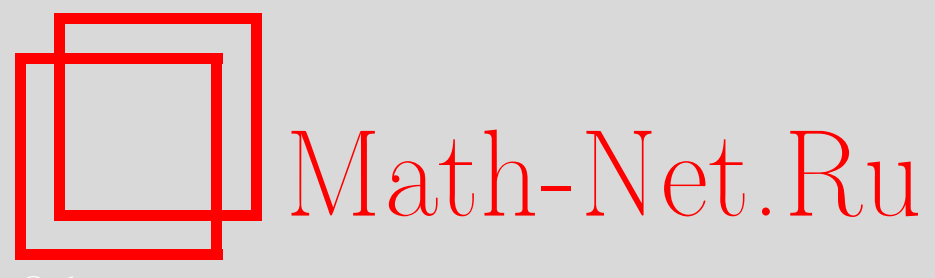

С. А. Степанов, Полиномиальные инварианты конечных групп над полями простой характеристики, Дискрет. матем., 1999, том 11, выпуск 3, 3-14

DOI: https://doi.org/10.4213/dm388

Использование Общероссийского математического портала Math-Net.Ru подразумевает, что вы прочитали и согласны с пользовательским соглашением http: //www.mathnet.ru/rus/agreement

Параметры загрузки:

IP : 3.85 .183 .62

26 апреля 2023 г., 14:40:51

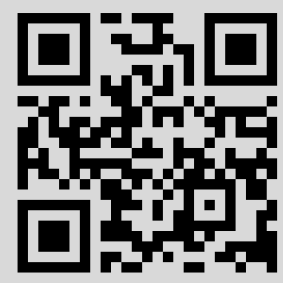




\title{
Полиномиальные инварианты конечных групп над полями простой характеристики
}

(c) 1999 г. $\quad$ C. A. Степанов

\begin{abstract}
Пусть $R$ - коммутативное кольцо с единичным элементом 1 и $G=S_{n}-$ симметрическая группа степени $n \geqslant 1$. Пусть $A_{m n}^{G}$ обозначает подалгебру инвариантов алгебры полиномов $A_{m n}=R\left[x_{11}, \ldots, x_{1 n} ; \ldots ; x_{m 1}, \ldots, x_{m n}\right]$ относительно $G$. Из классического результата Нетер следует, что если каждое целое число, не равное нулю, обратимо в $R$, то $A_{m n}^{G}$ порождается поляризованными элементарными симметрическими полиномами. Как недавно было показано Ричменом, этот результат остается в силе при условии, что $n$ ! обратимо в $R$. Цель настоящей статьи дать короткое доказательство результата Ричмена, основанное на использовании формулы Варинга и тесно связанное с первоначальным доказательством Нетер.

Работа выполнена при поддержке Университета Билкента, Анкара, Турция.
\end{abstract}

\section{1. Введение}

Пусть $m, n$ - положительные целые числа, $R$ - коммутативное кольцо с единичным элементом 1 и

$$
A_{m n}=R\left[x_{11}, \ldots, x_{m 1} ; \ldots ; x_{1 n}, \ldots, x_{m n}\right]
$$

- алгебра полиномов от $m n$ переменных $x_{i j}$ над $R$. Симметрическая группа $G=S_{n}$ действует на алгебре $A_{m n}$ как группа $R$-автоморфизмов по правилу

$$
g x_{i j}=x_{i, g(j)}, \quad g \in G .
$$

Обозначим $A_{m n}^{G}$ подалгебру инвариантов алгебры $A_{m n}$ относительно группы $G$ и определим поляризованные элементарные симметрические полиномы $u_{r_{1}, \ldots, r_{m}} \in A_{m n}^{G}$ от $n$ векторных переменных

$$
\left(x_{11}, \ldots, x_{m 1}\right), \ldots,\left(x_{1 n}, \ldots, x_{m n}\right)
$$

с помощью формального равенства

$$
\prod_{j=1}^{n}\left(1+x_{1 j} z_{1}+\ldots+x_{m j} z_{m}\right)=1+\sum_{1 \leqslant r_{1}+\ldots+r_{m} \leqslant n} u_{r_{1}, \ldots, r_{m}} z_{1}^{r_{1}} \ldots z_{m}{ }^{r_{m}}
$$

Если $R$ - Нетериан, то из теоремы Гильберта-Нетер $[4,6]$ следует, что $A_{m n}^{G}$ - конечно порожденная коммутативная $R$-алгебра и $A_{m n}$ конечно порождаема как 
модуль над $A_{m n}^{G}$. Более того, каждое целое число обратимо в $R$, инварианты $u_{r_{1}, \ldots, r_{m}}$ составляют полную систему образующих алгебры $A_{m n}^{G}$ над $R$ (см. [1], стр.9; [2], стр. $62 ;$ [14], стр. 37). Другими словами, каждый элемент $u$ алгебры $A_{m n}^{G}$ может быть записан в виде полинома от $u_{r_{1}, \ldots, r_{m}}, 1 \leqslant r_{1}+\ldots+r_{m} \leqslant n$, с коэффициентами из $R$. Приведенная выше система, порождающая инварианты, содержит $\left(\begin{array}{c}m+n \\ m\end{array}\right)-1$ элементов, связанных между собой различными алгебраическими соотношениями (см. [3], стр. 68 и [12]). Этот результат недавно был обобщен Ричменом [8] следующим образом.

Теорема 1. Пусть $G=S_{n}$ u $n$ ! обратимо в $R$. Тогда $A_{m n}^{G}$ порождается как $R$-алгебра поляризованными элементарными симметрическими полиномами

$$
u_{r_{1}, \ldots, r_{m}}, \quad 1 \leqslant r_{1}+\ldots+r_{m} \leqslant n
$$

степени, не большей $n$.

В частности, если $R$-- поле простой характеристики $p>n$, то $n$ ! обратимо в $R$, и мы приходим к следующему результату.

Следствие 1. Пусть $R-$ поле $u G=S_{n}$. Если характеристика $R$ равна нулю или $p>n$, где $p$ - простое число, то $A_{m n}^{G}$ порождается как $R$-алгебра поляризованнъми элементарными симметрическими полиномами $u_{r_{1}, \ldots, r_{m}}, 1 \leqslant r_{1}+\ldots+r_{m} \leqslant n$.

В этой статье мы приводим простое короткое доказательство теоремы 1 , основанное на поляризации классической формулы Варинга и тесно связанное с одним из двух первоначальных доказательств Нетер для случая, когда $R$ - поле характеристики 0. Несколько примеров, приведенных в заключительном разделе статьи показывают, что нельзя отказаться от ограничений на $R$, наложенных в теореме 1 .

Более обще, пусть $A=R\left[x_{1}, \ldots, x_{m}\right]$ - конечно порождаемая коммутативная $R$-алгебра, $G$ - конечная группа $R$-алгебры автоморфизмов $A$ и $A^{G}$ - подалгебра инвариантов группы $G$. Если $z_{1}, \ldots, z_{m}$ - коммутирующие переменные, то положим

$$
F\left(z_{1}, \ldots, z_{m}\right)=\prod_{\tau \in G}\left(1+\tau\left(x_{1}\right) z_{1}+\tau\left(x_{2}\right) z_{2}+\ldots+\tau\left(x_{m}\right) z_{m}\right) .
$$

Если каждое целое число, отличное от нуля, обратимо в $R$, то из теоремы Нетер следует, что $A^{G}$ порождается как $R$-алгебра коэффициентами $F\left(z_{1}, \ldots, z_{m}\right)$. Результат теоремы 1 и стандартные аргументы, основанные на использовании оператора Рейнольдса и отображения Нетер (см. [6]; [10], стр.63; [14], стр. 275), приводят к следующей теореме.

Tеорема 2. Eсли $|G|$ ! обратимо в $R$, то $A^{G}$ порождается как $R$-алгебра коэффициентами $F\left(z_{1}, \ldots, z_{m}\right)$. Другими словами, $A^{G}$ порождается над $R$ инвариантными полиномами от $x_{1}, \ldots, x_{m}$ степени, не большей $|G|$.

Этот результат приводит к эффективному алгоритму для вычисления полной системы порождающих полиномиальных инвариантов при условии, что $|G|$ ! обратимо в $R$. Существует другое конструктивное доказательство теоремы 1 , основанное на других аргументах и также восходящее к Нетер (см. [9] и [10], стр. 29). Верхняя граница степеней множества порождающих полиномов для алгебры инвариантов, приведенная в теореме 3, известна как граница Нетер (см. [9]; [10], стр. 28 и [11]). В последнем разделе статьи будет показано, что условия теоремы 3 не могут быть 
ослаблены. В частности, будет показано, что граница Нетер неверна, если $R$ - поле характеристики 2 и $G=S_{2}$. Другие результаты и задачи теории полиномиальных инвариантов в случае простой характеристики можно найти в [9] и [11].

\section{2. Порождающие инварианты симметрической группы}

Пусть $G=S_{n}$ - симметрическая группа степени $n \geqslant 1$, действующая на $R$-алгебре $A_{m n}=R\left[x_{11}, \ldots, x_{m 1} ; \ldots ; x_{1 n}, \ldots, x_{m n}\right]$ как группа $R$-автоморфизмов, $A_{m n}^{G}$ - подалгебра инвариантов $G$ в $A_{m n}$ и $u_{r_{1}, \ldots, r_{m}}, 1 \leqslant r_{1}+\ldots+r_{m} \leqslant n,-$ поляризованные элементарные симметрические полиномы в $A_{m n}^{G}$.

Пусть $v_{\sigma_{1}, \ldots, \sigma_{m}}-$ инвариантный полином в $A_{m n}^{G}$ вида

$$
v_{\sigma_{1}, \ldots, \sigma_{m}}=\sum_{j=1}^{n} x_{1 j}^{\sigma_{1}} \ldots x_{m j}^{\sigma_{m}}
$$

Если $m=1$, то согласно известной формуле Варинга (см. [13], стр. 13 и [2], стр.99)

$$
v_{\sigma}=\sum_{j=1}^{n} x_{j}^{\sigma}=\sum_{s_{1}+2 s_{2}+\ldots+n s_{n}=\sigma} c\left(s_{1}, \ldots, s_{n}\right) u_{1}^{s_{1}} \ldots u_{n}^{s_{n}},
$$

где $c_{s_{1}, \ldots, c_{n}}$ - целые числа вида

$$
c\left(s_{1}, \ldots, s_{n}\right)=(-1)^{s_{2}+2 s_{3}+\ldots+(n-1) s_{n}} \frac{\sigma\left(s_{1}+\ldots+s_{n}-1\right) !}{s_{1} ! \ldots s_{n} !} .
$$

Следующий результат можно рассматривать как обобщение формулы Варинга на случай, когда $m>1$ (см. также [12]).

Предложение 1. Пустъ $\sigma_{1}, \ldots, \sigma_{m}$ - неотрицательные челые числа,

$$
v_{\sigma_{1}, \ldots, \sigma_{m}}=\sum_{j=1}^{n} x_{1 j}^{\sigma_{1}} \ldots x_{m j}^{\sigma_{m}}
$$

- полином из $A_{m n}^{G}$ степени $\sigma=\sigma_{1}+\ldots+\sigma_{m} u u_{r_{1}, \ldots, r_{m}}, 1 \leqslant r_{1}+\ldots+r_{m-} \leqslant n,-$ поляризованный элементарный симметрический полином от векторов $\left(x_{1 j}, \ldots, x_{m j}\right)$, $1 \leqslant j \leqslant n$. Пусть $s_{1}, \ldots, s_{n}$ и $s_{1 \nu}, \ldots, s_{m \nu}$ - неотрицателъные целые числа такие, что $s_{1}+2 s_{2}+\ldots+n s_{n}=\sigma u s_{1 \nu}+\ldots+s_{m \nu}=\nu s_{\nu} \partial \Omega \Re 1 \leqslant \nu \leqslant n$, nусть также

$$
w_{s_{1 \nu}, \ldots, s_{m \nu}}=\sum \frac{s_{\nu} !}{\sigma_{\nu 1} ! \ldots \sigma_{\nu s_{\nu}} !} \prod_{\tau=1}^{s_{\nu}} u_{r_{1 \tau}, \ldots, r_{m \tau}}^{\sigma_{\nu \tau}},
$$

где суммирование проводится по всем неотрицательным целым числам

$$
r_{1 \tau}, \ldots, r_{m s_{\nu}}, \quad \sigma_{\nu 1}, \ldots, \sigma_{\nu s_{\nu}}
$$

таким, что

$$
\begin{aligned}
r_{\mu 1} \sigma_{\nu 1}+\ldots+r_{m s_{\nu}} \sigma_{\nu s_{\nu}} & =s_{\mu \nu}, \sigma_{\nu 1}+\ldots+\sigma_{\nu s_{\nu}}=s_{\nu} \\
r_{1 \tau}+\ldots+r_{m \tau} & =\nu, \quad 1 \leqslant \mu \leqslant m, \quad 1 \leqslant \nu \leqslant n, \quad 1 \leqslant \tau \leqslant s_{\nu} .
\end{aligned}
$$


Тогда

$$
v_{\sigma_{1}, \ldots, \sigma_{m}}=\frac{\sigma_{1} ! \ldots \sigma_{m} !}{\sigma !} \sum_{s_{1}+2 s_{2}+\ldots+n s_{n}=\sigma} c\left(s_{1}, \ldots, s_{n}\right) \sum \prod_{i=1}^{n} v_{s_{1 \nu_{i}}, \ldots, s_{m \nu_{i}}}
$$

где внутренняя сумма берется по всем неотрицателъным целым числам

$$
s_{\mu \nu_{1}}, \ldots, s_{\mu \nu_{n}}
$$

таким, что

$$
\begin{aligned}
s_{\mu \nu_{1}}+\ldots+s_{\mu \nu_{n}} & =\sigma_{\mu}, \\
s_{1 \nu_{i}}+\ldots+s_{m \nu_{i}} & =i s_{i}, \quad 1 \leqslant \mu \leqslant m, \quad 1 \leqslant i \leqslant n .
\end{aligned}
$$

Доказательство. Положим в (2)

$$
x_{j}=x_{1 j} z_{1}+\ldots+x_{m j} z_{m}, \quad 1 \leqslant j \leqslant m .
$$

Поскольку

$$
\left(x_{1 j} z_{1}+\ldots+x_{m j} z_{m}\right)^{\sigma}=\sum_{\sigma_{1}+\ldots+\sigma_{m}=\sigma} \frac{\sigma !}{\sigma_{1} ! \ldots \sigma_{m} !} x_{1 j}^{\sigma_{1}} \ldots x_{m j}^{\sigma_{m}} z_{1}^{\sigma_{1}} \ldots z_{m}^{\sigma_{m}}
$$

справедливо равенство

$$
\sum_{j=1}^{n}\left(x_{1 j} z_{1}+\ldots+x_{m j} z_{m}\right)^{\sigma}=\sum_{\sigma_{1}+\ldots+\sigma_{m}=\sigma} \frac{\sigma !}{\sigma_{1} ! \ldots \sigma_{m} !}\left(\sum_{j=1}^{n} x_{1 j}^{\sigma_{1}} \ldots x_{m j}^{\sigma_{m}}\right) z_{1}^{\sigma_{1}} \ldots z_{m}^{\sigma_{m}} .
$$

С другой стороны,

$$
\sum_{1 \leqslant j_{1}<\ldots<j_{\nu} \leqslant n} \prod_{s=1}^{\nu}\left(x_{1 j_{s}} z_{1}+\ldots+x_{m j_{s}} z_{m}\right)=\sum_{r_{1}+\ldots+r_{m}=\nu} u_{r_{1}, \ldots, r_{m}} z_{1}^{r_{1}} \ldots z_{m}^{r_{m}}
$$

и, следовательно, с учетом (2),

$$
\begin{aligned}
\sum_{j=1}^{n}\left(x_{1 j} z_{1}+\ldots+x_{m j} z_{m}\right)^{\sigma} & \\
& =\sum_{s_{1}+2 s_{2}+\ldots+n s_{n}=\sigma} c\left(s_{1}, \ldots, s_{n}\right) \prod_{\nu=1}^{n}\left(\sum_{r_{1}+\ldots+r_{m}=\nu} u_{r_{1}, \ldots, r_{m}} z_{1}^{r_{1}} \ldots z_{m}^{r_{m}}\right)^{s_{\nu}} .
\end{aligned}
$$

В результате получаем, что

$$
\begin{aligned}
\sum_{\sigma_{1}+\ldots+\sigma_{m}=\sigma} & \frac{\sigma !}{\sigma_{1} ! \ldots \sigma_{m} !}\left(\sum_{j=1}^{n} x_{1 j}^{\sigma_{1}} \ldots x_{m j}^{\sigma_{m}}\right) z_{1}^{\sigma_{1}} \ldots z_{m}^{\sigma_{m}} \\
& =\sum_{s_{1}+s l_{2}+\ldots+n s_{n}=\sigma} c\left(s_{1}, \ldots, s_{n}\right) \prod_{\nu=1}^{n}\left(\sum_{r_{1}+\ldots+r m=\nu} u_{r_{1}, \ldots, r_{m}} z_{1}^{r_{1}} \ldots z_{m}^{r_{m}}\right)^{s_{\nu}} .
\end{aligned}
$$


Ясно, что

$$
\left(\sum_{r_{1}+\ldots+r_{m}=\nu} u_{r_{1}, \ldots, r_{m}} z_{1}^{r_{1}} \ldots z_{m}^{r_{m}}\right)^{s_{v}}=\sum_{s_{1 \nu}+\ldots+s_{m \nu}=\nu s_{\nu}} w_{s_{1 \nu}, \ldots, s_{m \nu}} z_{1}^{s_{1 \nu}} \ldots z_{m}^{s_{m \nu}}
$$

где

$$
w_{s_{1 \nu}, \ldots, s_{m \nu}}=\sum \frac{s_{\nu} !}{\sigma_{\nu 1} ! \ldots \sigma_{\nu s_{\nu}} !} \prod_{\tau=1}^{s_{\nu}} u_{r_{1 \tau}, \ldots, r_{m \tau}}^{\sigma_{\nu \tau}}
$$

и суммирование ведется по всем целым неотрицательным

$$
\sigma_{\nu 1}, \ldots, \sigma_{\nu s_{\nu}}, \quad r_{1 \tau}, \ldots, r_{m \tau}, \quad 1 \leqslant \tau \leqslant s_{\nu}
$$

таким, что

$$
\begin{aligned}
r_{\mu 1} \sigma_{\nu 1}+\ldots+r_{\mu s_{\nu}} \sigma_{\nu s_{\nu}} & =s_{\nu \tau}, & & 1 \leqslant \mu \leqslant m \\
r_{1 \tau}+\ldots+r_{m \tau} & =\nu, & & 1 \leqslant \tau \leqslant s_{\nu} \\
\sigma_{\nu 1}+\ldots+\sigma_{\nu s_{\nu}} & =s_{\nu} . & &
\end{aligned}
$$

Отсюда следует, что

$$
\begin{aligned}
& \sum_{\sigma_{1}+\ldots+\sigma_{m}=\sigma}\left(\sum_{j=1}^{n} x_{1 j}^{\sigma_{1}} \ldots x_{m j}^{\sigma_{m}}\right) z_{1}^{\sigma_{1}} \ldots z_{m}^{\sigma_{m}} \\
& \quad=\sum_{\sigma_{1}+\ldots+\sigma_{m}=\sigma}\left(\sum_{s_{1}+2 s_{2}+\ldots+n s_{n}=\sigma} c\left(s_{1}, \ldots, s_{n}\right) \sum_{S} \prod_{i=1}^{n} w_{s_{1 \nu_{i}}, \ldots, s_{m \nu_{i}}}\right) z_{1}^{\sigma_{1}} \ldots z_{m}^{\sigma_{m}},
\end{aligned}
$$

где вторая внутренняя сумма берется по множеству $S$ всех целых неотрицательных $s_{\mu \nu_{1}}, \ldots, s_{\mu \nu_{n}}, \leqslant \mu \leqslant m$, таких, что

$$
\begin{aligned}
s_{\mu \nu_{1}}+\ldots+s_{\mu \nu_{n}}=\sigma_{\mu}, & & 1 \leqslant \mu \leqslant m \\
s_{1 \nu_{i}}+\ldots+s_{m \nu_{i}}=i s_{i}, & & 1 \leqslant i \leqslant n .
\end{aligned}
$$

Таким образом, мы приходим к равенству

$$
\sum_{j=1}^{n} x_{1 j}^{\sigma_{1}} \ldots x_{m j}^{\sigma_{m}}=\frac{\sigma_{1} ! \ldots \sigma_{m} !}{\sigma !} \sum_{s_{1}+2 s_{2}+\ldots+n s_{n}=\sigma} c\left(s_{1}, \ldots, s_{n}\right) \sum_{S} \prod_{i=1}^{n} w_{s_{1 \nu_{i}}, \ldots, s_{m \nu_{i}}},
$$

где множество $S$ определено в предыдущей формуле. Это равенство доказывает теорему.

Если $\sigma=\sigma_{1}+\ldots+\sigma_{m} \leqslant n+1$, то

$$
v_{\sigma_{1}, \ldots, \sigma_{m}}=\frac{\sigma_{1} ! \ldots \sigma_{m} !}{\sigma !} \sum_{s_{1}+2 s_{2}+\ldots+n s_{n}=\sigma} c\left(s_{1}, \ldots, \sigma_{m}\right) \prod_{i=1}^{n} v_{s_{1 \nu_{i}}, \ldots, s_{m \nu_{i}}}
$$

содержит только поляризованные элементарные симметрические полиномы

$$
u_{r_{1}, \ldots, r_{m}}, \quad 1 \leqslant r_{1}+\ldots+r_{m} \leqslant n .
$$

Кроме того, коэффициенты полинома $v_{\sigma_{1}, \ldots, \sigma_{m}}$ являются рациональными числами со знаменателями, не делящимися ни на одно простое число $p>\sigma$. Из этого замечания вытекает следующее утверждение. 
Следствие 2. Если число $n$ ! обратимо в $R u \sigma=\sigma_{1}+\ldots+\sigma_{m} \leqslant n+1$, mo

$$
v_{\sigma_{1}, \ldots, \sigma_{m}}=\sum_{j=1}^{n} x_{1 j}^{\sigma_{1}} \ldots x_{m j}^{\sigma_{m}}
$$

есть полином над $R$ от поляризованных элементарных симметрических полиномов $u_{r_{1}, \ldots, r_{m}}, 1 \leqslant r_{1}+\ldots+r_{m} \leqslant n$, степени, не большей $n$.

Покажем теперь, что любой инвариант из $A_{m n}^{G}$ можно представить как полином над $R$ от $v_{\sigma_{1}, \ldots, \sigma_{m}}$.

Предложение 2. Пусть $f-$ одночлен из $A_{m n} u$

$$
v=\sum_{u \in\{\tau(f) \mid \tau \in G\}} u .
$$

Тогда $v$ - полином над $R$ от инвариантов

$$
v_{\sigma_{1}, \ldots, \sigma_{m}}=\sum_{j=1}^{n} x_{1 j}^{\sigma_{1}} \ldots x_{m j}^{\sigma_{m}}
$$

где $\sigma_{1}, \ldots, \sigma_{m}$ - неотрицательные челье числа такие, что $0 \leqslant \sigma_{1}+\ldots+\sigma_{m} \leqslant \operatorname{deg} f$.

Доказательство. Запишем $f$ в виде $f=f_{1} \ldots f_{n}$, где каждый из множителей $f_{j}$ есть одночлен в $R\left[x_{1 j}, \ldots, x_{m j}\right]$. Положим

$$
d(f)=\max _{1 \leqslant j \leqslant n} \operatorname{deg} f_{j}
$$

и докажем утверждение индукцией по $\delta(f)=\operatorname{deg} f-d(f)$. Предположим вначале, что $\delta(f)=0$. Тогда

$$
f=f_{j}=x_{1 j}^{\alpha_{1}} \ldots x_{m j}^{\alpha_{m}}
$$

для некоторого $j \in\{1,2, \ldots, n\}$ и $\left(\alpha_{1}, \ldots, \alpha_{m}\right), 0 \leqslant \alpha_{1}+\ldots+\alpha_{m} \leqslant \operatorname{deg} f$, поэтому

$$
v=\sum_{u \in\{\tau(f) \mid \tau \in G\}} u=\sum_{j=1}^{n} x_{1 j}^{\alpha_{1}} \ldots x_{m j}^{\alpha_{m}}
$$

Предположим теперь, что $\delta(f)>0$ и пусть $j \in\{1,2, \ldots, n\}$ таково, что $d(f)=$ $\operatorname{deg} f_{j}<\operatorname{deg} f$. Определим $v_{j}$ и $v_{j}^{\prime}$, полагая

$$
v_{j}=\sum_{u \in\left\{\tau\left(f_{j}\right) \mid \tau \in G\right\}} u, \quad v_{j}^{\prime}=\sum_{u^{\prime} \in\left\{\tau\left(f / f_{j}\right) \mid \sigma \in G\right\}} u^{\prime} .
$$

По предположению индукции $v_{j}$ и $v_{j}^{\prime}$ являются полиномами от

$$
v_{\sigma_{1}, \ldots, \sigma_{m}}, \quad 0 \leqslant \sigma_{1}+\ldots+\sigma_{m} \leqslant \operatorname{deg} f
$$

Для каждого $\rho \in G$ определим $U_{\rho}$ как множество всех пар $\left(u, u^{\prime}\right)$ таких, что

$$
u \in\left\{\tau\left(f_{j}\right) \mid \tau \in G\right\}, \quad u^{\prime} \in\left\{\tau\left(f / f_{j}\right) \mid \tau \in G\right\}, \quad u u^{\prime}=\rho(f),
$$


и заметим, что отображение

$$
U_{i d} \rightarrow U_{\rho}, \quad\left(u, u^{\prime}\right) \rightarrow\left(\tau(u), \tau\left(u^{\prime}\right)\right)
$$

есть биекция. Таким образом, $\left|U_{\rho}\right|=\left|U_{i d}\right|$ для всех $\rho \in G$. Заметим также, что $d\left(u u^{\prime}\right) \geqslant d(f)$ для всех $u \in\left\{\tau\left(f_{j}\right) \mid \tau \in G\right\}$ и $u^{\prime} \in\left\{\tau\left(f / f_{j}\right) \mid \tau \in G\right\}$, причем равенство достигается тогда и только тогда, когда $u u^{\prime} \in\{\tau(f) \mid \tau \in G\}$. Поэтому

$$
v_{j} v_{j}^{\prime}=\left|U_{i d}\right| \sum_{u \in\{\tau(f) \mid \sigma \in G\}} u+\sum_{f^{\prime}: \operatorname{deg} f^{\prime}=\operatorname{deg} f, d\left(f^{\prime}\right)>d(f)} \sum_{u \in\left\{\tau\left(f^{\prime}\right) \mid \tau \in G\right\}} u .
$$

По предположению индукции, инвариант

$$
v_{j} v_{j}^{\prime}-\left|U_{i d}\right| \sum_{u \in\{\tau(f) \mid \sigma \in G\}} u=\sum_{f^{\prime}: \operatorname{deg} f^{\prime}=\operatorname{deg} f, d\left(f^{\prime}\right)>d(f)} \sum_{u \in\left\{\tau\left(f^{\prime}\right) \mid \tau \in G\right\}} u .
$$

есть полином над $R$ от $v_{\sigma_{1}, \ldots, \sigma_{m}}, 0 \leqslant \sigma_{1}+\ldots+\sigma_{m} \leqslant \operatorname{deg} f$. Мощность $U_{i d}$ не превосходит мощности $\left\{\tau\left(f_{j}\right) \mid \tau \in G\right\}$, которая в свою очередь не превосходит мощности $\left\{x_{1 j}^{\sigma_{1}} \ldots x_{m j}^{\sigma_{m}} \mid 1 \leqslant j \leqslant n\right\}$, так что $1 \leqslant\left|U_{i d}\right| \leqslant n$. Так как число $n$ ! обратимо в $R$, мы заключаем, что

$$
\sum_{u \in\{\tau(f) \mid \tau \in G\}} u
$$

есть полином над $R$ от $v_{\sigma_{1}, \ldots, \sigma_{m}}, 0 \leqslant \sigma_{1}+\ldots+\sigma_{m} \leqslant \operatorname{deg} f$. Это завершает доказательство.

\section{3. Доказательство теоремы 1}

Пусть $G=S_{n}$ - симметрическая группа степени $n$. Предположим, что $f$ есть одночлен в $A_{m n}$ и $w \in A_{m n}^{G}$ есть полиномиальный инвариант $G$. Так как $\tau(w)=w$ для любого $\tau \in G$, полиномы $w$ и $\tau(w)$ имеют одинаковые коэффициенты. Это показывает, что каждый полиномиальный инвариант $G$ есть $R$-линейная комбинация инвариантов

$$
v=\sum_{u \in\{\tau(f) \mid \tau \in G\}} u,
$$

где $f$ пробегает значения одночленов, появляющихся в $w$.

Пусть $\left(i_{1}, i_{2}, \ldots, i_{\mu}\right)$ - последовательность элементов $i_{1}, \ldots, i_{\mu} \in\{1, \ldots, n\}$. Вначале докажем, что каждый инвариант $w_{\mu}$ вида

$$
w_{\mu}=\sum_{j=1}^{n} x_{i_{1}, j} \ldots x_{i_{\mu}, j}
$$

есть полином над $R$ от поляризованных элементарных симметрических полиномов

$$
u_{r_{1}, \ldots, r_{m}}, \quad 1 \leqslant r_{1}+\ldots+r_{m} \leqslant n .
$$

Если $\mu \leqslant n+1$, это утверждение следует из предложения 1 . Предположим теперь, что $\mu>n+1$, и проведем доказательство индукцией по $\mu$. Для $j=1, \ldots, n$ положим

$$
\tilde{x}_{i_{s}, j}= \begin{cases}x_{i_{s}, j}, & \text { если } s \leqslant n, \\ x_{i_{n+1}, j} x_{i_{n+2}, j} \ldots x_{i_{\mu+1}, j}, & \text { если } s=n+1,\end{cases}
$$


и рассмотрим

$$
w_{\mu+1}=\sum_{j=1}^{n} \tilde{x}_{i_{1}, j} \ldots \tilde{x}_{i_{n}, j} \tilde{x}_{i_{n+1}, j} .
$$

Пусть $\tilde{A}_{m n}=R\left[\tilde{x}_{11}, \ldots, \tilde{x}_{m 1} ; \ldots ; \tilde{x}_{1 n}, \ldots, \tilde{x}_{m n}\right]$, и $\tilde{A}_{m n}^{G}-$ подалгебра инвариантов из $\tilde{A}_{m n}$. Из следствия 2 следует, что $w_{\mu+1}-$ полином над $R$ от поляризованных элементарных симметрических полиномов

$$
\tilde{u}_{r_{1}, \ldots, r_{m}} \in \tilde{A}_{m n}, \quad 1 \leqslant r_{1}+\ldots+r_{m} \leqslant n .
$$

Поскольку каждый полином $\tilde{u}_{r_{1}, \ldots, r_{m}}$ имеет вид

$$
\tilde{u}_{r_{1}, \ldots, r_{m}}=\sum_{\tilde{u} \in\{\tau(\tilde{f}) \mid \tau \in G\}} \tilde{u}
$$

при некотором одночлене $\tilde{f} \in \tilde{A}_{m n}$ степени, не большей $n$, в силу предложения 2 он может быть записан как полином над $R$ от инвариантов

$$
\tilde{v}_{\sigma_{1}, \ldots, \sigma_{m}}=\sum_{j=1}^{n} \tilde{x}_{1 j}^{\sigma_{1}} \ldots \tilde{x}_{m j}^{\sigma_{m}}
$$

степени, не большей $n$. Поэтому

$$
\tilde{v}_{\sigma_{1}, \ldots, \sigma_{m}}=\sum_{j=1}^{n} x_{1 j}^{s_{1}} \ldots x_{m j}^{s_{j}},
$$

где $1 \leqslant s_{1}+\ldots+s_{m} \leqslant \mu$. Согласно предположению индукции каждый инвариант $\tilde{v}_{\sigma_{1}, \ldots, \sigma_{m}}$ есть полином над $R$ от $u_{r_{1}, \ldots, r_{m}}, 1 \leqslant r_{1}+\ldots+r_{m} \leqslant n$, так что $w_{\mu+1}-$ также полином над $R$ от поляризованных элементарных симметрических полиномов $u_{r_{1}, \ldots, r_{m}}, 1 \leqslant r_{1}+\ldots+r_{m} \leqslant n$.

Для завершения доказательства заметим, что каждый элемент $v \in A_{m n}^{G}$ может быть записан, в силу предложения 2 , как полином над $R$ от инвариантов $w_{\mu}$.

\section{4. Примеры}

Пример 1. Пусть $m=3$ и $n=2$. Покажем, что кубический полином

$$
v_{111}=x_{11} x_{21} x_{31}+x_{12} x_{22} x_{32}
$$

инвариантный относительно группы $G=S_{2}$, не может быть представлен как полином от инвариантов $u_{100}, u_{010}, u_{001}, u_{200}, u_{110}, u_{101}, u_{020}, u_{011}, u_{002}$ над $F_{2}$, простым полем характеристики 2. По предложению 1

$$
2 v_{111}=2 u_{100} u_{010} u_{001}-\left(u_{100} u_{011}+u_{010} u_{101}+u_{001} u_{110}\right),
$$

и поскольку образующие $u_{100}, u_{010}, u_{001}, u_{110}, u_{101}, u_{011}$ алгебры

$$
\mathbf{Q}\left[x_{11}, x_{21}, x_{31} ; x_{12}, x_{22}, x_{32}\right]^{G}
$$


алгебраически независимы над $\mathbf{Q}$, это разложение единственно. Следовательно, $v_{111}$ нельзя представить над $F_{2}$ как полином от

$$
u_{100}, u_{010}, u_{001}, u_{200}, u_{110}, u_{101}, u_{020}, u_{011}, u_{002} \text {. }
$$

Таким образом, граница Нетер неверна, если характеристика равна 2. Поэтому условия теорем 1 и 2 нельзя ослабить. Кроме того, мы видим, что

$$
u_{100}, u_{010}, u_{001}, u_{011}, u_{101}, u_{110}
$$

алгебраически зависимы над $F_{2}$.

Покажем теперь, что полином $f_{\sigma_{1} \sigma_{2} \sigma_{3}}$ вида

$$
f_{\sigma_{1} \sigma_{2} \sigma_{3}}=x_{11}^{\sigma_{1}} x_{21}^{\sigma_{2}} x_{31}^{\sigma_{31}}+x_{12}^{\sigma_{1}} x_{22}^{\sigma_{2}} x_{32}^{\sigma_{3}}
$$

может быть представлен как полином от

$$
u_{001}, u_{010}, u_{100}, u_{002}, u_{011}, u_{020}, u_{101}, u_{110}, u_{200}
$$

и $v_{111}$ с целыми коэффициентами.

Сначала покажем, что любой полином

$$
f_{\sigma_{1} \sigma_{2}}=x_{11}^{\sigma_{1}} x_{21}^{\sigma_{2}}+x_{12}^{\sigma_{2}} x_{22}^{\sigma_{2}}
$$

есть полином от $u_{01}, u_{10}, u_{02}, u_{11}, u_{20}$ над Z. Действительно, справедливы равенства

$$
x_{11}+x_{12}=u_{10}, \quad x_{21}+x_{22}=u_{01}
$$

И

$$
x_{11}^{2}+x_{12}^{2}=u_{10}^{2}-2 u_{20}, \quad x_{11} x_{21}+x_{12} x_{22}=u_{01} u_{10}-u_{11}, \quad x_{21}^{2}+x_{22}^{2}=u_{01}^{2}-2 u_{02} .
$$

При $\sigma=\sigma_{1}+\sigma_{2}>2$ без потери общности можно считать, что $\sigma_{2} \geqslant 2$. В таком случае

$$
\begin{aligned}
f_{\sigma_{1} \sigma_{2}} & =\left(x_{21}+x_{22}\right) f_{\sigma_{1} \sigma_{2}-1}-\left(x_{11}^{\sigma_{11}} x_{21}^{\sigma_{2}-1} x_{22}+x_{21}^{\sigma_{1}} x_{22}^{\sigma_{2}-1} x_{21}\right) \\
& =\left(x_{21}+x_{22}\right) f_{\sigma_{1} \sigma_{2}-1}-x_{21} x_{22} f_{\sigma_{1}, \sigma_{2}-2}=u_{01} f_{\sigma_{1} \sigma_{2}-1}-u_{02} f_{\sigma_{1} \sigma_{2}-2}
\end{aligned}
$$

и мы можем использовать индукцию по двум параметрам $m$ и $\sigma$.

Аналогично, при $\sigma=\sigma_{1}+\sigma_{2}+\sigma_{3}>3$ без потери общности можно считать, что $\sigma_{3} \geqslant 2$. В этом случае

$$
\begin{aligned}
f_{\sigma_{1} \sigma_{2} \sigma_{3}} & =\left(x_{31}+x_{32}\right) f_{\sigma_{1} \sigma_{2} \sigma_{3}-1}-\left(x_{11}^{\sigma_{1}} x_{21}^{\sigma_{2}} x_{31}^{\sigma_{3}-1} x_{32}+x_{21}^{\sigma_{1}} x_{22}^{\sigma_{2}} x_{32}^{\sigma_{3}-1} x_{31}\right) \\
& =u_{001} f_{\sigma_{1}, \sigma_{2}, \sigma_{3}-1}-u_{002} f_{\sigma_{1} \sigma_{2} \sigma_{3}-2}
\end{aligned}
$$

и утверждение получается с использованием индукции по $m$ и $\sigma$.

Пример 2. Пусть $m=4$ и $n=2$. Покажем, что многочлен четвертой степени

$$
v_{1111}=x_{11} x_{21} x_{31} x_{41}+x_{12} x_{22} x_{32} x_{42},
$$

инвариантный относительно группы $G=S_{2}$, не может быть представлен над $F_{2}$ как полином от

$$
u_{r_{1}, r_{2}, r_{3}, r_{4}}, \quad 1 \leqslant r_{1}+r_{2}+r_{3}+r_{4} \leqslant 4
$$


Предположим противное, пусть

$$
\begin{aligned}
v_{1111}=a & u_{1000} u_{0100} u_{0010} u_{0001} \\
& +b\left(u_{1000} u_{0100} u_{0011}+u_{1000} u_{0010} u_{0101}+u_{1000} u_{0001} u_{0110}\right) \\
& +b\left(u_{0100} u_{0001} u_{1010}+u_{0100} u_{0010} u_{1001}+u_{0010} u_{0001} u_{1100}\right) \\
& +c\left(u_{1100} u_{0011}+u_{1010} u_{0101}+u_{1001} u_{0110}\right)
\end{aligned}
$$

где $a, b, c \in F_{2}$. Заметим, что

$$
\begin{aligned}
& u_{1000}=x_{11}+x_{21}, \quad u_{0100}=x_{21}+x_{22}, \\
& u_{0010}=x_{31}+x_{32}, \quad x_{0001}=x_{41}+x_{42}, \\
& u_{1100}=x_{11} x_{22}+x_{12} x_{12}, \\
& u_{1010}=x_{11} x_{32}+x_{12} x_{31}, \\
& u_{1001}=x_{11} x_{42}+x_{12} x_{41}, \\
& u_{0110}=x_{21} x_{32}+x_{22} x_{31}, \\
& u_{0101}=x_{21} x_{42}+x_{22} x_{41}, \\
& u_{0011}=x_{31} x_{42}+x_{32} x_{41} .
\end{aligned}
$$

Дифференцируя эти равенства по $x_{11}$ и принимая во внимание, что

$$
\frac{\partial u_{1000}}{\partial x_{11}}=1, \quad \frac{\partial u_{0100}}{\partial x_{11}}=0, \quad \frac{\partial u_{0010}}{\partial x_{11}}=0, \quad \frac{\partial u_{0001}}{\partial x_{11}}=0
$$

И

$$
\begin{array}{lll}
\frac{\partial u_{1100}}{\partial x_{11}}=x_{22}, & \frac{\partial u_{1010}}{\partial x_{11}}=x_{32}, & \frac{\partial u_{1001}}{\partial x_{11}}=x_{42}, \\
\frac{\partial u_{0110}}{\partial x_{11}}=0, & \frac{\partial u_{0101}}{\partial x_{11}}=0, & \frac{\partial u_{0011}}{\partial x_{11}}=0,
\end{array}
$$

получаем, что

$$
\begin{aligned}
x_{21} x_{31} x_{41}=a & u_{0100} u_{0010} u_{0001}+b\left(u_{0100} u_{0011}+u_{0010} u_{0101}+u_{0001} u_{0110}\right) \\
& +b\left(u_{0100} u_{0001} x_{32}+u_{0100} u_{0010} x_{42}+u_{0010} u_{0001} x_{22}\right) \\
& +c\left(u_{0011} x_{22}+u_{0101} x_{32}+u_{0110} x_{42}\right) .
\end{aligned}
$$

Полагая теперь в последнем равенстве

$$
x_{21}=x_{31}=x_{41}=x_{12}=x_{22}=x_{32}=x_{42}=1 \text {, }
$$

приходим к равенству $1=8 a+24 b+6 c$, которое невозможно в $F_{2}$.

Наконец, из предложения 2 следует, что

$$
\begin{aligned}
6 v_{1111}=6 & u_{1000} u_{0100} u_{0010} u_{0001} \\
& -2\left(u_{1000} u_{0100} u_{0011}+u_{1000} u_{0010} u_{0101}+u_{1000} u_{0001} u_{0110}\right) \\
& -2\left(u_{0100} u_{0001} u_{1010}+u_{0100} u_{0010} u_{1001}+u_{0010} u_{0001} u_{1100}\right) \\
& +\left(u_{1100} u_{0011}+u_{1010} u_{0101}+u_{1001} u_{0110}\right)
\end{aligned}
$$

так что полиномы $u_{1100}, u_{1010}, u_{1001}, u_{0110}, u_{0101}, u_{0011}$ алгебраически зависимы над $F_{2}$. С другой стороны, эти полиномы алгебраически независимы над $\mathbf{Q}$. 
Пример 3. Предыдущий пример можно обобщить следующим образом. Пусть $m$ - целое число, $m>n \geqslant 2$, и $p$ - простой делитель $n$. Пусть $F_{p}$ - простое конечное поле характеристики $p>0$ и

$$
v_{11 \ldots 11}=\sum_{j=1}^{n} x_{1 j} \ldots x_{m j}
$$

- однородный полином степени $m$. Покажем, что полином $v_{11 \ldots 11}$ нельзя представить как полином от инвариантов

$$
u_{r_{1}, \ldots, u_{m}}, \quad 1 \leqslant r_{1}+\ldots r_{m} \leqslant n
$$

над полем $F_{p}$.

Предположим противное, пусть при некоторых $a_{s_{1}, \ldots, s_{n}} \in F_{p}$

$$
v_{11 \ldots 11}=\sum_{s_{1}+2 s_{2}+\ldots+n s_{n}=m} a_{s_{1}, \ldots, s_{n}} \sum_{R} \prod_{\nu=1}^{n} \prod_{\sigma_{\nu}=1}^{s_{\nu}} u_{r_{1 \sigma_{\nu}}, \ldots, r_{m \sigma_{\nu}}}
$$

где суммирование во второй сумме проводится по множеству $R$ всех целых неотрицательных

$$
r_{1 \sigma_{\nu}}, \ldots, r_{m \sigma_{\nu}}, \quad 1 \leqslant \nu \leqslant n
$$

таких, что

$$
\begin{aligned}
r_{1 \sigma_{\nu}}+\ldots+r_{m \sigma_{\nu}} & =\nu, \quad 1 \leqslant \sigma_{\nu} \leqslant s_{\nu}, \quad 1 \leqslant \nu \leqslant n \\
r_{i \sigma_{1}}+\ldots+r_{i \sigma_{n}} & =1, \quad 1 \leqslant i \leqslant m .
\end{aligned}
$$

Без потери общности предположим, что если $k \leqslant n-$ наименьшее положительное целое число такое, что $s_{k} \geqslant 1$, то

$$
r_{1 \sigma_{k}}= \begin{cases}1, & \text { если } \sigma_{k}=1, \\ 0, & \text { если } 2 \leqslant \sigma_{k} \leqslant s_{k} .\end{cases}
$$

Дифференцируя приведенное выше равенство по $x_{11}$ и учитывая, что

$$
\frac{\partial u_{r_{1}, \ldots, r_{m}}}{\partial x_{11}}= \begin{cases}0, & \text { если } r_{1}=0 \\ u_{0, r_{2}, \ldots, r_{m}}^{(1,0, \ldots, 0)}, & \text { если } r_{1}=1\end{cases}
$$

где $u_{0, r_{2}, \ldots, r_{m}}^{(1,0, \ldots, 0)}-$ соответствующий элементарный симметрический полином от векторов $\left(x_{2 j}, \ldots, x_{m j}\right), 1 \leqslant j \leqslant n$, получаем, что

$$
x_{21} \ldots x_{m 1}=\sum_{s_{1}+2 s_{2}+\ldots+n s_{n}=m} a_{s_{1}, \ldots, s_{n}} \sum_{j=1}^{n} \Psi_{s_{1}, \ldots, s_{n}}^{(j)},
$$

где

$$
\Psi_{s_{1}, \ldots, s_{n}}^{(j)}=\sum_{R} \prod_{\sigma_{k}=2}^{s_{k}} u_{0, r_{2 \sigma_{k}}, \ldots, r_{m \sigma_{k}}}\left(\prod_{\substack{\nu=1 \\ \nu \neq k}}^{n} \prod_{\sigma_{\nu}=1}^{s_{\nu}} u_{0, r_{2 \sigma_{\nu}} \ldots, r_{m \sigma_{\nu}}}\right)
$$


и множество $R$ определено выше. Обозначим $\omega_{0, r_{2}, \ldots, r_{m}}$ значение $u_{0, r_{2}, \ldots, r_{m}}$ в точке

$$
\left(x_{11}, \ldots, x_{m 1} ; \ldots, ; x_{1 n}, \ldots, x_{m n}\right)=(1, \ldots, 1 ; \ldots ; 1 \ldots, 1) .
$$

Поскольку $m>n \geqslant 2$, каждая двоичная последовательность $\left(0, r_{2}, \ldots, r_{m}\right)$, встречающаяся в последнем равенстве, содержит $l$ ненулевых элементов при некотором $1 \leqslant l \leqslant n$. В этом случае

$$
\omega_{0, r_{2}, \ldots, r_{m}}=n(n-1) \ldots(n-l+1)
$$

и полагая в (4)

$$
x_{11}=\ldots=x_{m 1}=\ldots=x_{1 n}=\ldots=x_{m n}=1,
$$

получаем равенство

$$
1=n \sum_{s_{1}+2 s_{2}+\ldots+n s_{n}=m} b_{s_{1}, \ldots, s_{n}},
$$

которое невозможно в $F_{p}$ для любого простого $p$, делящего $n$.

\section{Список литературы}

1. Benson D. J., Polynomial Invariants of Finite Groups. Cambr. Univ. Press, Cambridge, 1993.

2. Bourbaki N., Elements of Mathematics, Algebra II. Springer, Berlin, 1990.

3. Dieudonne J. A., Carrel J. B., Invariant Theory, Old and New. Academic Press, New York, 1971.

4. Hilbert D., Über die vollen Invariantensystem. Math. Ann. (1893) 42, 313-373.

5. Mumford D., Geometric Invariant Theory. Springer, Berlin, 1993.

6. Noether E., Der Endlichkeitssatz der Invarianten endlicher Gruppen. Math. Ann. (1916) 77, 89-92.

7. Noether E., Der Endlichkeitssatz der Invarianten endlicher linear Gruppen der Characteristik p. Nachr. v. d. Ges. d. Wiss. zu Göttingen (1926), 28-35.

8. Richman D. R., Explicit generators of the invariants of finite groups. Adv. Math. (1996) 124, 49-76.

9. Schmid B. J., Finite Groups and Invariant Theory. Lect. Notes Math. (1991) 1478.

10. Smith L., Polynomial Invariants of Finite Groups. A. K. Peters, Wellesley, MA, 1995.

11. Smith L., Polynomial invariants of finite groups. A survey of recent developments. Bull. Amer. Math. Soc. (1997) 34, 211-250.

12. Stepanov S. A., On vector invariants of the symmetric group. Discrete Math. Appl. (1996) 6, 135-147.

13. Waring E., Meditationes Algebraicae. Cambr. Univ. Press, Cambridge, 1782.

14. Weyl H., The Classical Groups. Inst. Adv. Study, Princeton, 1946.

Статья поступила 25.05.1999. 